Check for updates

Cite this: RSC Adv., 2019, 9, 28876

Received 18th June 2019

Accepted 30th August 2019

DOI: $10.1039 / c 9 r a 04558 a$

rsc.li/rsc-advances

\section{Anisotropic nanocomposite films of hydroxypropylcellulose and graphene oxide with multi-responsiveness $\dagger$}

\author{
Zhimin Ying, ${ }^{\mathrm{a}}$ Xiao Ying Lin, ${ }^{\mathrm{b}}$ Cong Du, ${ }^{\text {b }}$ Si Yu Zheng, ${ }^{\mathrm{b}}$ Zi Liang Wu (D) *b \\ and Qiang Zheng ${ }^{b}$
}

Anisotropic nanocomposite films of hydroxypropylcellulose (HPC) and graphene oxide (GO) were fabricated by blade-coating of the aqueous mixture to align the substance and subsequent solvent evaporation to freeze the oriented structure. Owing to the anisotropic structure, the composite films showed anisotropic mechanical properties and response to external stimuli. The influences of GO content, stretch rate, and relative humidity on the anisotropic structure and mechanical properties of the films were investigated. The incorporation of GO did not destroy the anisotropic structure of the HPC film, but improved the mechanical properties to some extent and favoured the bending deformation and locomotion of the composite film under the humidity gradient. These behaviours were associated with the large aspect ratio and excellent gas barrier property of GO nanosheets that favoured suppressing the slippage of HPC chains and enhanced the differential volume change at the top and bottom surfaces of the film. The composite HPC film with GO or reduced GO also responded to near-infrared light due to the photothermal effect and the variation of HPC matrix at a high temperature. This facile strategy should be applicable to other natural or synthetic polymers to fabricate anisotropic composite films with potential applications as optical devices, sensors, and actuators.

\section{Introduction}

Cellulose, as the most abundant natural polymer, has been receiving increasing attention owing to its renewability, low cost, and biodegradability. ${ }^{1}$ Myriad functional cellulose-based materials, including advanced densified woods and regenerated cellulose materials, have been developed with promising applications in textiles, water treatment, tissue engineering, flexible electronics, and so forth..$^{2-4}$ Especially, the new dissolution and regeneration technology and surface modification strategies greatly improve the processability and solubility, and enrich the functionalities. Besides the cellulose nanocrystals and nanofibrils, ${ }^{5-9}$ cellulose derivatives have tailored functions by molecular modification and can be dispersed in solvents or other polymers at the molecular level. ${ }^{10}$

Hydroxypropylcellulose (HPC) is a water-soluble derivative of cellulose. The aqueous solution of HPC shows a liquid

${ }^{a}$ Second Affiliated Hospital, School of Medicine, Zhejiang University, Hangzhou 310009, China

${ }^{b}$ Ministry of Education Key Laboratory of Macromolecular Synthesis and Functionalization, Department of Polymer Science and Engineering, Zhejiang University, Hangzhou 310027, China. E-mail: congdu@zju.edu.cn; wuziliang@zju. edu.cn

$\dagger$ Electronic supplementary information (ESI) available. See DOI: $10.1039 / \mathrm{c} 9 \mathrm{ra} 04558 \mathrm{a}$ crystalline (LC) phase when its concentration is above a critical low concentration, $C_{\mathrm{LC}}^{*}$, of $\sim 40 \mathrm{wt} \% .^{11}$ The HPC solution in LC phase with multi-domain alignments can be oriented into monodomain by mechanical shear. The oriented structure of HPC molecules can be fixed by subsequent chemical reaction to form a network ${ }^{12}$ or solvent evaporation to form a casted film to suppress to structural relaxation of HPC chains..$^{13}$ Anisotropic HPC films were fabricated by blade-coating the LC solution and solvent evaporation at a low humidity environment; the resultant film showed anisotropic mechanical properties and response to the change of relative humidity. ${ }^{14}$

Other components can be combined into the blade-coated HPC films to afford improved properties and/or additional functions. To avoid the destruction of LC phase of HPC solution, the additive with the capacity to form a LC structure is preferred. For example, Fernandes et al. incorporated cellulose nanocrystals (NCs) to the LC solution of HPC to prepare composite anisotropic films with tunable structural colors and enhanced mechanical properties. ${ }^{15}$ Carbon nanotube (CNT) was also incorporated to fabricate composite HPC films. Echeverria et al. found that the addition of $0.01 \mathrm{wt} \% \mathrm{CNT}$ led to reinforcement of the anisotropic film with an increase in the Young's modulus by a factor of 1.9 to 2.6, respectively, when the film was elongated parallel or perpendicular to the orientation of HPC molecules. ${ }^{16}$ 
Beside the 1D additives, graphene oxide (GO) has geometry feature of $2 \mathrm{D}$ nanosheet with a large aspect ratio, and its aqueous suspension shows LC phase with a very low $C_{\mathrm{LC}}^{*}$, of $\sim 0.025$ wt $\%{ }^{17}$ Therefore, GO should be incorporated into HPC solution to prepare anisotropic composite films. Although there are no reports on GO-HPC films, GO has been often used to prepare anisotropic composites with excellent mechanical performances, ${ }^{18-22}$ gas barrier properties, ${ }^{23}$ and response to humidity gradient and near-infrared (NIR) light. ${ }^{24-29}$

In this paper, we incorporate GO into HPC solution and prepare the anisotropic composite film by blade-coating. Polarizing optical microscope, atomic force microscope, and tensile tests were adopted to examine the topographical features and anisotropic properties of the composite films. The influences of stretch rate, relative humidity, and GO content on the anisotropic mechanical properties of the films were examined. It was found that the incorporation of GO did not destroy the anisotropic structure of the HPC films, but improved the mechanical properties to some extent and favoured the bending deformation and locomotion of the composite film under humidity gradients. After reducing GO of the composite film into reduced GO (RGO) by UV light irradiation, the anisotropic RGO-HPC film showed irreversible response to NIR light due to the structural rearrangement and volume expansion of the HPC matrices at high temperature. These anisotropic composite films with specific mechanical properties and multi-responsiveness should find applications as sensors and actuators.

\section{Experimental}

\subsection{Materials}

HPC with a weight-average molecular weight of $1.0 \times 10^{5}$ and molar substitution of 3.5 was purchased from Sigma-Aldrich. Aqueous graphene oxide (GO) solution $\left(1.2 \mathrm{mg} \mathrm{mL}^{-1}\right)$ was purchased from Hangzhou Gaoxi Technology Co. Ltd. The monodispersed GO sheets had a lateral width of 5-20 $\mu \mathrm{m} .{ }^{30}$ Silica gel was purchased from Sinopharm Chemical Reagent Co., Ltd. Milli-Q deionized water $(18.2 \mathrm{M} \Omega \mathrm{cm})$ was used in all experiments.

\subsection{Preparation of anisotropic HPC films with and without GO}

Aqueous HPC solution with concentration of $50 \mathrm{wt} \%$ was prepared by dissolving prescribed amount of HPC in water, according to the protocol described elsewhere. ${ }^{4}$ To prepare the mixture solutions of HPC and GO, prescribed amounts (Table S1 $\dagger$ ) of GO solution (1.2 $\mathrm{mg} \mathrm{mL}^{-1}$ ), HPC powder, and water were added into a glass vessel, which were kept in a $4{ }^{\circ} \mathrm{C}$ refrigerator and stirred every day for 2 weeks. ${ }^{31} \mathrm{GO}-\mathrm{HPC}$ solutions were blade-coated on a flat polyimide film (thickness of $0.125 \mathrm{~mm}$ ) at room temperature and $40 \%$ environmental relative humidity (RH), by using an XT-300 coating machine (Shijiazhuang Oschina Mechanical Technology Co., Ltd) with a shear rate of $100 \mathrm{~s}^{-1}$. GO-HPC composite films were fabricated by subsequent solvent evaporation in a chamber with $40 \% \mathrm{RH}$ for $12 \mathrm{~h}$. Silica gels were adopted to adjust the humidity of the chamber. The composite films are coded as GH- $x-y$, where $x$ and $y$ are the content in wt\% of GO and HPC in the mixture solution, respectively. Anisotropic HPC films without GO were fabricated by blade coating the $50 \mathrm{wt} \%$ HPC solution following the same procedure. The resultant HPC films are coded as H-50.

With environmental $\mathrm{RH}$ of $35 \%$, GO-HPC composite films were cut into $60 \mathrm{~mm} \times 5 \mathrm{~mm}$ rectangles from directions perpendicular and parallel to the shear direction and then peeled off from the polyimide substrate. The film thickness measured by a screw micrometer was $30 \mu \mathrm{m}$ for tensile tests and $10 \mu \mathrm{m}$ for morphological tests. RGO-HPC films were fabricated by exposing GO-HPC films to $254 \mathrm{~nm}$ UV light irradiation for $8 \mathrm{~h}$ to reduce GO to reduced graphene oxide (RGO). Corresponding composite films were coded as RH- $x-y$.

\subsection{Characterizations}

Polarizing optical microscope and AFM observation. The birefringence of the mixture solutions with different GO content was observed under a polarizing optical microscope (POM, DSFi2, Nikon) with insertion of a $530 \mathrm{~nm}$ tint plate. The band textures of GO-HPC composite films with different film thicknesses and fabrication shear rates were observed with magnifications $\times 40$ and $\times 100$. The surface morphology of the GOHPC composite films was characterized using an atomic force microscope (AFM, MFP-3D).

UV-vis spectroscopy. UV-vis absorption spectra of GO-HPC films reduced by $254 \mathrm{~nm}$ UV light irradiation for different time were obtained using a UV-vis spectrophotometer (UV-1800, Shimadzu).

Photothermal effect characterization. The temperature variations of $\mathrm{H}-50$ and GH-0.06-50, as well as RH-0.06-50 films reduced by $254 \mathrm{~nm}$ UV light for $8 \mathrm{~h}, 24 \mathrm{~h}$, and $54 \mathrm{~h}$, under the irradiation of $808 \mathrm{~nm}$ near-IR (NIR) light with different output power were monitored by using a thermal imager (FLIR E60). The temperature variations of the composite films after switching on and off the near infrared light with different output power were also monitored. The NIR light was generated by a diode laser (LSR $808 \mathrm{H}-7 \mathrm{~W}$, Lasever).

Tensile tests. The mechanical properties of GO-HPC composite films were measured by a tensile tester (Instron 3343) at room temperature. The dimensions of the test sample were $60 \mathrm{~mm} \times 5 \mathrm{~mm} \times 30 \mu \mathrm{m}$, and the gauge length was $20 \mathrm{~mm}$. Without specific notification, the tests were carried out at $35 \%$ $\mathrm{RH}$ with a stretch rate of $80 \mathrm{~mm} \mathrm{~min}^{-1}$. The mechanical parameters including the yield stress $\left(\sigma_{\mathrm{y}}\right)$ and Young's modulus $(E)$ were averaged from six parallel measurements. $E$ was calculated from the slope of the initial linear regime of stressstrain curves.

Stress relaxation tests. Stress relaxation of HPC, GO-HPC, and RGO-HPC films with different compositions was measured after applying a strain of 3\%. During the tests, cyclic variation of the humidity between $35 \%$ and $60 \%$ or switching on and off of the near infrared light $\left(70 \mathrm{~mW} \mathrm{~m}^{-2}\right)$ was applied to films to investigate the response of the films.

\section{Results and discussion}

As shown in Fig. 1a, 50 wt\% HPC solution showed strong birefringence, indicating the formation of liquid crystalline (LC) 

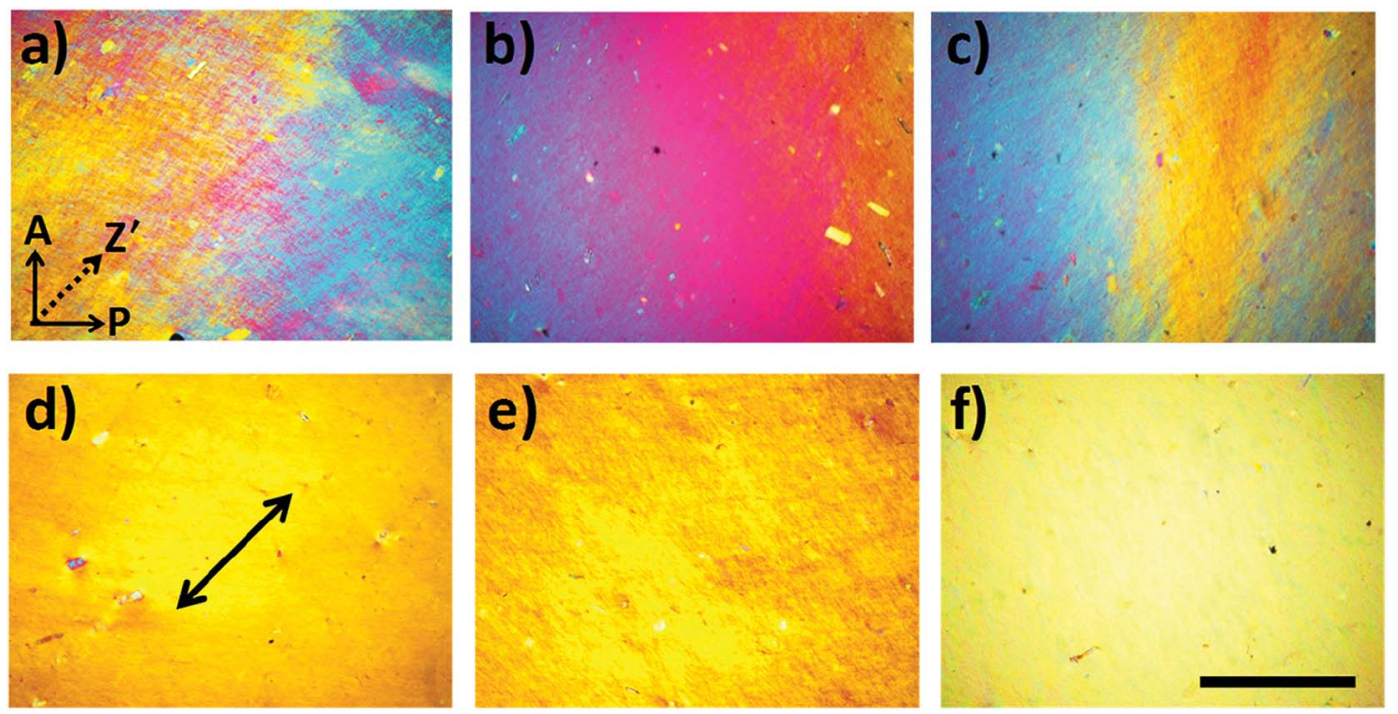

Fig. 1 POM images of the mixture solutions with $50 \mathrm{wt} \%$ HPC and different content of GO before (a-c) and after (d-f) applying mechanical shear. GO content: $0 \mathrm{wt} \%$ ( $\mathrm{a}$ and d), $0.03 \mathrm{wt} \%$ (b and e), and $0.06 \mathrm{wt} \%$ ( $\mathrm{c}$ and $\mathrm{f}$ ). The sample thickness is $0.5 \mathrm{~mm}$ and the shear rate is $100 \mathrm{~s}^{-1}$. The black double arrow indicates the shear direction. Scale bar: $300 \mu \mathrm{m}$. A: analyzer; P: polarizer; Z': slow axis of the tint plate.

phase, despite of lacking long-range orientation. In the presence of a small amount of GO, the birefringence and corresponding LC phase were well maintained. On the contrary, the size of uniform domain with a specific director in the mixture solution slightly increased after the incorporation of $0.03 \mathrm{wt} \%$ and $0.06 \mathrm{wt} \% \mathrm{GO}$ (Fig. $1 \mathrm{~b}$ and c). This may be attributed to the following reasons: (i) aqueous GO solution also shows a LC phase with a very low critical concentration, $C_{\mathrm{LC}}^{*}$ of $\sim 0.025 \mathrm{wt} \%$, due to the large aspect ratio of the $2 \mathrm{D}$ nanosheets and their $\pi-\pi$ stacking interaction, ${ }^{17}$ and (ii) the lateral dimension of GO is much larger than the size of HPC molecule, favouring the formation of large sized LC domains in the mixture solution. After shearing, the birefringent color became uniform, indicating the transformation from multi-domain to monodomain of the LC phase (Fig. 1d-f). The birefringent color of the sheared LC solution was brighter in the presence of GO. These results indicated that the incorporation of GO did not damage the LC structure of HPC but benefited the shear orientation of HPC. GO did not act as impurities to destroy the LC phase, but it improved the shear orientation of HPC.

Anisotropic casted films were obtained by placing the bladecoated GO-HPC solutions in a chamber with relatively low humidity (RH of $40 \%$ ) to evaporate the solvent. Although the structures may partially relax during the solvent evaporation, the macroscopically anisotropic structure was well maintained in the dry films, as shown in Fig. $\mathrm{S} 1, \dagger$ because of the high a)
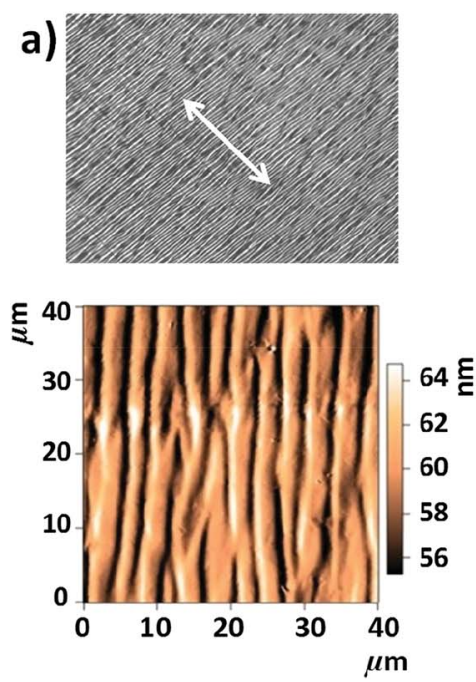

b)

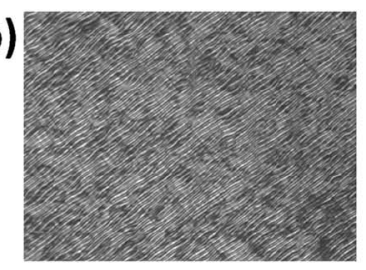

c)
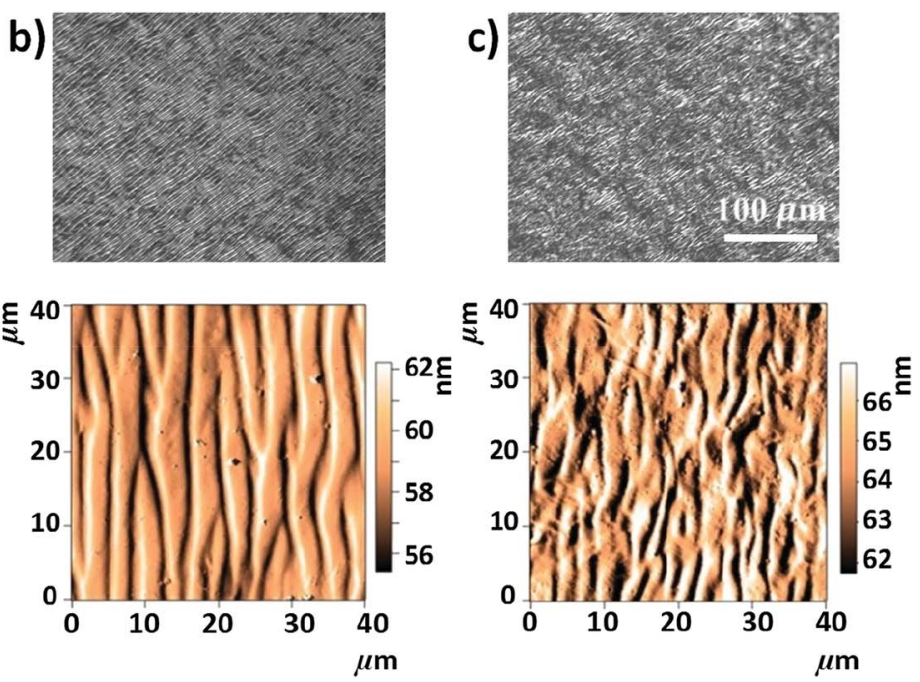

Fig. 2 POM (top) and AFM images (bottom) of composite film with 50 wt\% HPC and different content of GO: 0 wt\% (a), 0.016 wt\% (b), and $0.06 \mathrm{wt} \%$ (c). The casted film with a thickness of $\sim 10 \mu \mathrm{m}$ was prepared by blade-coating with a shear rate of $100 \mathrm{~s}^{-1}$. 
viscosity of the mixture solution that slowed the structural relaxation. The structural relaxation during the drying process was manifested by the formation of typical periodical band textures perpendicular to the shear direction (Fig. 2a). ${ }^{32,33} \mathrm{~A}$ systematic investigation found that a larger shear rate and a smaller film thickness resulted in a larger orientation degree and a more ordered band texture (Fig. S2 $\dagger$ ). HPC molecules were well orientated when the shear rate exceeded $3.2 \mathrm{~s}^{-1}$, and the band texture became more regular when the shear rate increased to $100 \mathrm{~s}^{-1}$. At a constant shear rate, increasing film thickness decreased the orientation degree of HPC molecules and the regularity of band texture. This was because the thicker film required longer evaporation time, which was not beneficial for freezing the oriented structure. ${ }^{34}$ As shown in Fig. $2 b$, the incorporation of a small amount of GO (the concentration was lower than the $C_{\mathrm{LC}}^{*}$ of $0.025 \mathrm{wt} \%$ ) had no evident influence on the orientation and band texture of the blade-coated film. However, further increase of GO content to $0.06 \mathrm{wt} \%$ led to a decrease in the regularity of band texture (Fig. 2c), because the large sized GO nanosheets with sufficient amount hindered the relaxation of HPC molecules. AFM images also confirmed this fact that, with increasing GO content, the bands became less regular, accompanying with a more blurred boundary. We should note that the molecular alignment of HPC was parallel to the shear direction, although the texture bands were perpendicular to the shear direction.

Tensile tests were performed to characterize the mechanical performances of the anisotropic HPC films with and without GO. Typical yielding was found in the stress-strain curves of the samples being stretched perpendicular or parallel to the direction of molecular alignment (Fig. 3). As expected, the yielding stress $\sigma_{\mathrm{y}}$ and Young's modulus $E$ increased with the stretch rate
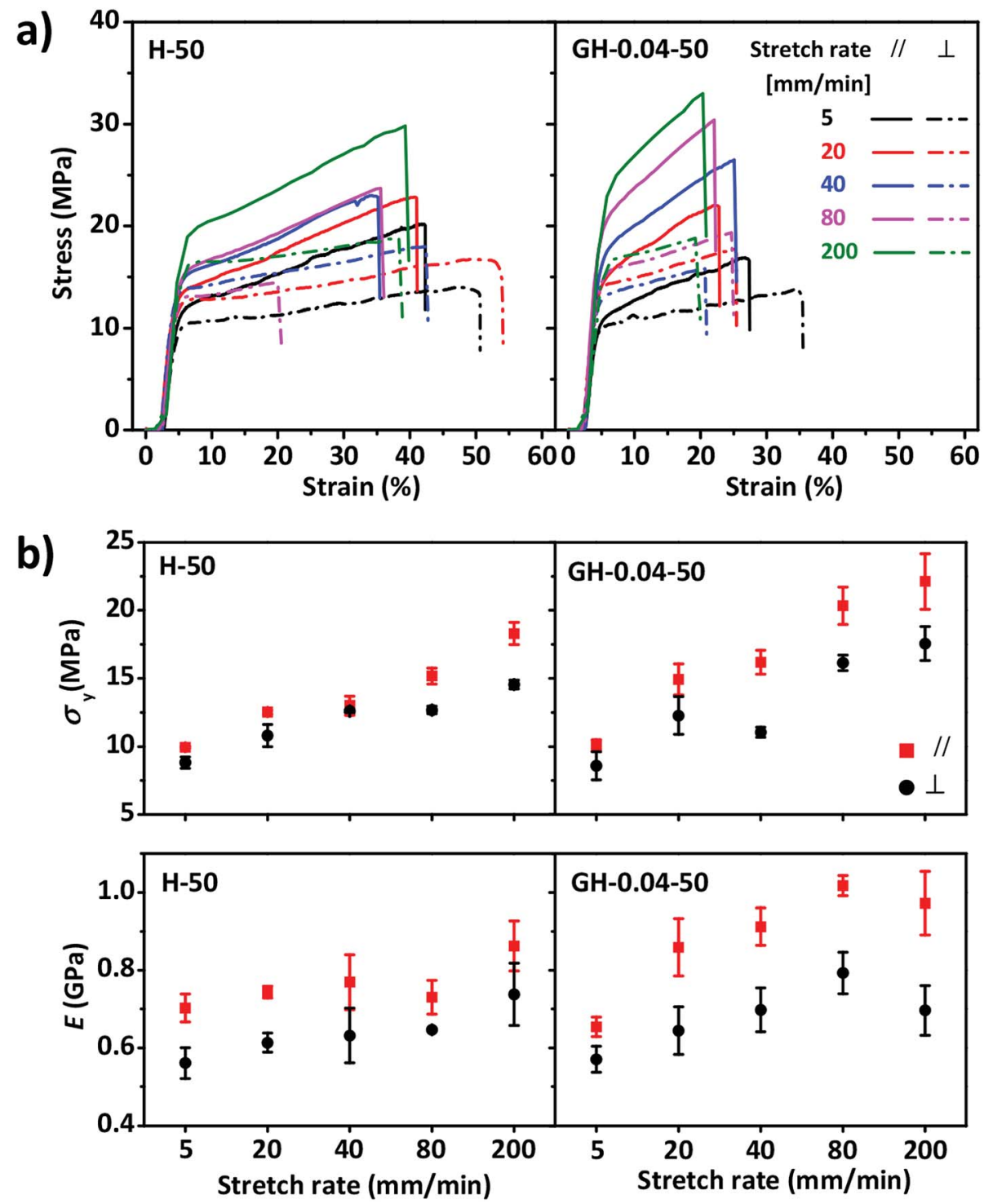

Fig. 3 Tensile stress-strain curves (a) and corresponding mechanical properties (b) of H-50 and GH-0.04-50 composite films at different stretch rates. The tests were performed at room temperature and constant relative humidity of $35 \%$. The films with thickness of $\sim 30 \mu \mathrm{m}$ were prepared by blade-coating with a shear rate of $100 \mathrm{~s}^{-1}$. 
a)

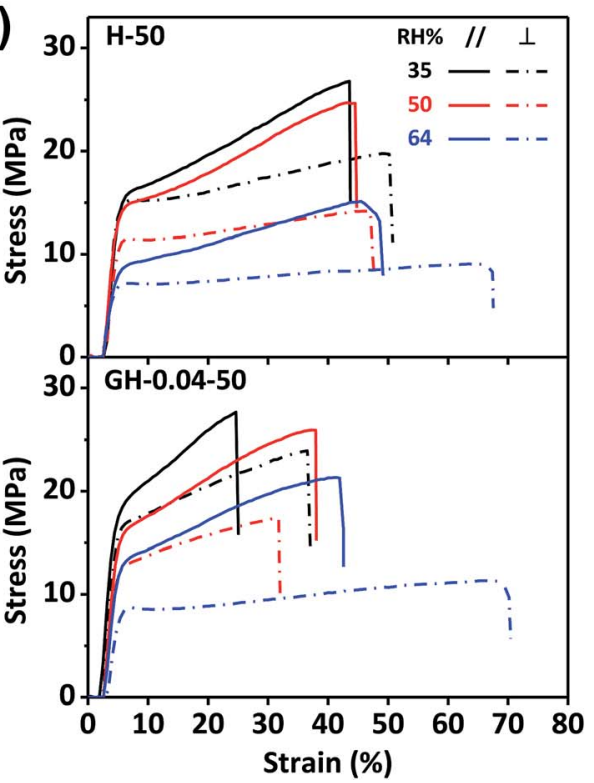

b)

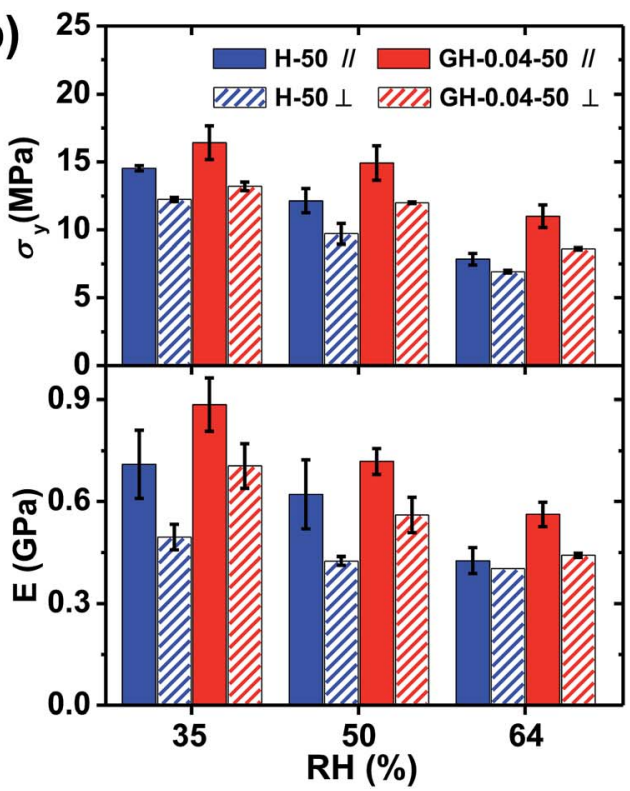

Fig. 4 Tensile stress-strain curves (a) and corresponding mechanical properties (b) of H-50 and GH-0.04-50 composite films at a stretch rate of $80 \mathrm{~mm} \mathrm{~min}^{-1}$ and different relative humidity.

without evident decrease in the breaking strain $\varepsilon_{\mathrm{b}}$. However, $\sigma_{\mathrm{y}}$ and $E$ of the samples being stretched parallel to the molecular alignment were larger than those perpendicular to molecular alignment, indicating the anisotropic mechanical properties. Strain stiffening became more evident in the GO-HPC composite film, when compared to the pure HPC film, probably because of the capacity of 2D GO nanosheets to efficiently suppress the chain sliding of HPC. Owing to the same reason, the composite film had relatively larger $\sigma_{\mathrm{y}}$ and $E$ yet smaller $\varepsilon_{\mathrm{b}}$ than that of pure HPC film. Considering the mechanical properties of the films and the repeatability of the tensile tests, stretch rate of $80 \mathrm{~mm} \mathrm{~min}^{-1}$ was selected in the following experiments.
The stress-strain curves of the $\mathrm{H}-50$ and $\mathrm{GH}-0.04-50$ films performed at different relative humidity (35\%, 50\%, and 64\%) were shown in Fig. 4. For both films, the breaking stress $\sigma_{\mathrm{b}}$, yielding stress $\sigma_{\mathrm{y}}$, yielding strain $\varepsilon_{\mathrm{y}}$, and Young's modulus $E$ decreased with increasing $\mathrm{RH}$, while the breaking strain $\varepsilon_{\mathrm{b}}$ increased with RH. However, the RH influenced the mechanical properties of HPC and GO-HPC films to different degree. For the pure HPC film, the anisotropicity of mechanical properties, determined by the difference of mechanical parameters measured at different tensile directions, became smaller with the increase in RH. At RH of 64\%, the HPC film showed similar stress-strain curves (before yielding) at different tensile directions, indicating the disappearance of mechanical anisotropy.
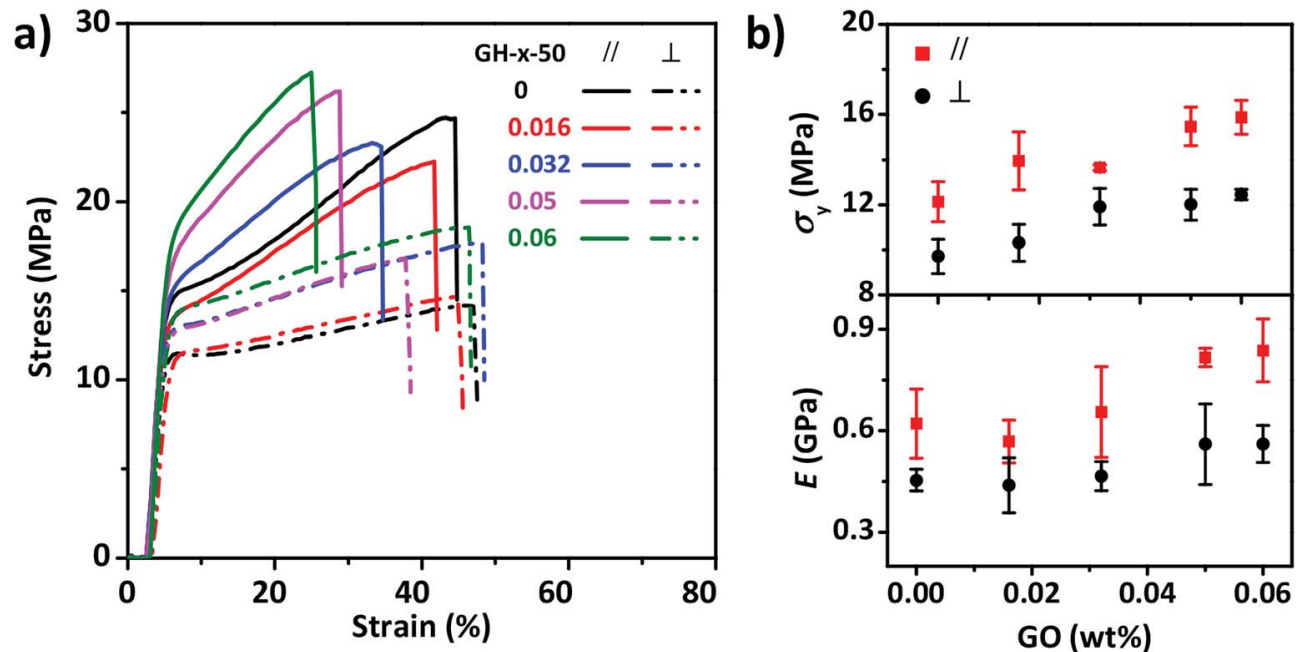

Fig. 5 Tensile stress-strain curves (a) and corresponding mechanical properties (b) of composite films with different content of GO at a stretch rate of $80 \mathrm{~mm} \mathrm{~min}^{-1}$ and relative humidity of $50 \%$. 
By contrast, the difference of mechanical properties of the GOHPC composite films between parallel and perpendicular directions was almost constant. The different behaviours resulted from different hydrophilicity between HPC and GO. HPC bearing a large amount of hydroxyl groups had strong capacity to absorb more water from the environment with relatively high $\mathrm{RH}$ (Fig. S3†), which plasticized the HPC molecules and softened the films. However, GO is less hydrophilic than HPC, and the lamellar structure of GO could enhance the resistant of HPC to water, resulting in a larger mechanical anisotropicity of GO-HPC films.

The mechanical properties of the anisotropic GO-HPC composite films were also influenced by the content of GO. As shown in Fig. 5, the films with different content of GO showed different stress-strain curves obtained at constant stretch rate ( $80 \mathrm{~mm} \mathrm{~min}^{-1}$ ) and RH (50\%). $E$ and $\sigma_{\mathrm{y}}$ of the composite films increased with the increasing GO content, indicating that GO nanosheets can reinforce the HPC film to some extent. GO content had different influences on the anisotropic mechanical properties of the composite films at different RH (Fig. S4 $\dagger$ ). When $\mathrm{RH}$ was $35 \%, E$ in the perpendicular and parallel direction both increased with GO content, while $\sigma_{\mathrm{y}}$ in the direction perpendicular to the molecular alignment kept constant and that in the parallel direction increased with GO content. When $\mathrm{RH}$ was $50 \%$ or $64 \%, \sigma_{\mathrm{y}}$ and $E$ in the parallel direction still increased with GO content but $E$ in the perpendicular direction became constant. When $\mathrm{RH}$ was equal to $64 \%$, it was clear that the anisotropicity of the films mechanical properties increased with GO content because the water resistant ability of GO prevented the drop of $\sigma_{\mathrm{y}}$ and $E$ in the parallel direction.

The response of the film to humidity gradient also resulted in programmed bending deformation. ${ }^{35,36}$ As shown in Fig. S5, $\uparrow$ when the anisotropic HPC film with the bottom surface (the side on polyimide during the casting) was up and placed atop the silica gels, the film bent upward with bending direction perpendicular to the molecular alignment (i.e. the shear direction). On the other hand, when the surface with periodic wrinkles (the side open to the air during the casting) was up, the film bend upward with bending direction parallel to the molecular alignment. When the humidity gradient diminished after removing the silica gels, the film returned to its original flat shape, indicating the reversible deformation by control the humidity gradient. The different bending direction relative to the molecular alignment was caused by the different structures at the two sides of the composite film. Since the oriented structure was fixed by solvent evaporation, HPC molecules at the top surface should have better alignment than the bottom surface. Thus, when the top surface with wrinkles was exposed to the silica gels with low $\mathrm{RH}$, anisotropic volume contraction was expected, where the volume shrank more perpendicular to the director. As a consequence, the film bent upward with bending direction perpendicular to the molecular alignment. By contrast, when the bottom surface was exposed to the environment with low $\mathrm{RH}$, the top surface exposed to relatively high $\mathrm{RH}$ of $70 \%$ (higher than the $\mathrm{RH}$ of the chamber during the drying process) should expand its volume more in the direction perpendicular to the director. This anisotropic volume expansion played a predominant role in the deformation, resulting in the bending parallel to the director.

When the anisotropic HPC film was exchanged to GO-HPC composite film, continuous locomotion was observed. As shown in Fig. 6, the composite film was placed atop the silica gel with the bottom surface exposed to high humidity, the film bent upward with the bending direction perpendicular to the molecular alignment due to the same reason as mentioned
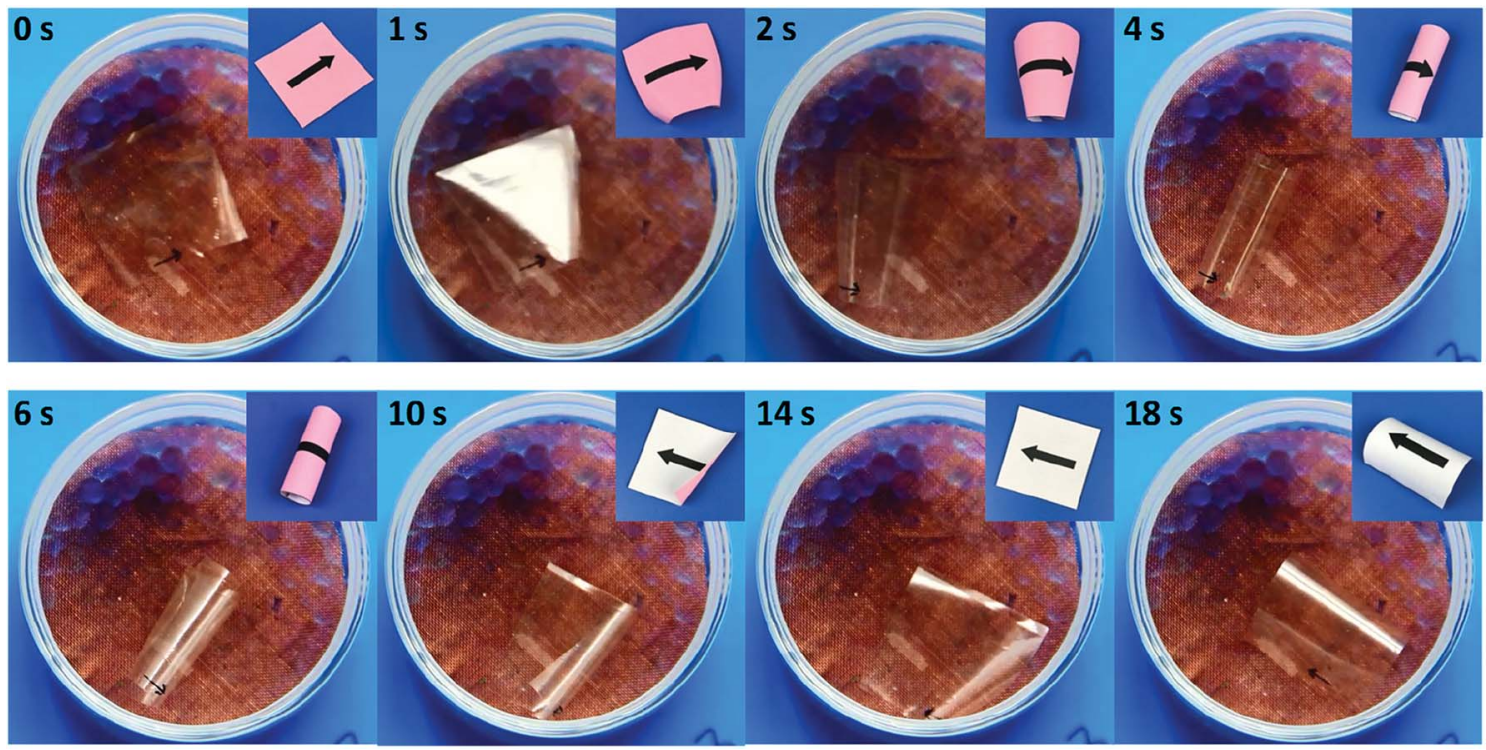

Fig. 6 Photos of the locomotion of a GH-0.016-50 film with a thickness of $10 \mu \mathrm{m}$ on a copper mesh (below RH: 40\%; above RH: $70 \%$ ). Insets show a piece of paper representing the different stages of the locomotion. The black arrow indicates the shear direction during the fabrication process of the film. The pink and white faces of the paper stand for the bottom and top surfaces of the composite film. 
above (2 s). Because of the high humidity gradient and small film thickness, the film quickly rolled (4 s) and flipped under the gravity ( $6 \mathrm{~s}) \cdot{ }^{37}$ As the film edges that originally contacted to the copper mesh swelled because they were exposed to the environment with higher humidity, and the section that was originally exposed to high humidity contacted to the copper mesh and shrank, the rolled film became flattened (14 s). The top surface of the film was now exposed to high humidity, leading to bending parallel to the director (18 s). The continuous bending and flipping resulted in locomotion of the anisotropic composite film (Movie $\mathrm{S} 1 \dagger$ ). This result suggested that the presence of GO favoured the bending deformation with large amplitude that caused the flipping and locomotion, when compared to the HPC film without GO. The reason might be related to the excellent gas barrier property ${ }^{23}$ of GO nanosheet that favours the built-up of different volume change at the top and bottom surfaces exposed to environment with different $\mathrm{RH}$ and thus the bending deformation with relatively high speed and amplitude.

To afford additional functions, GO in the composite films was reduced to RGO under the irradiation of $254 \mathrm{~nm}$ UV light. The reduction of GO to RGO in the composite film was confirmed by the appearance of the characteristic peak at $\sim 270 \mathrm{~nm}$ in the UV-vis absorption spectrum (Fig. S6†). The intensity of the peak increased with the UV irradiation time. However, exposing to the UV light also caused the decomposition of the HPC film. It was found that $\sigma_{\mathrm{y}}$ and $E$ of the composite film decreased by $40 \%$ and $52 \%$, respectively, after being exposed to UV light for $36 \mathrm{~h}$. Therefore, $8 \mathrm{~h}$ was selected as the reduction time.

The photothermal effect should render the composite RGOHPC film with photo responsiveness. ${ }^{38,39}$ The photothermal behaviours of $\mathrm{H}-50, \mathrm{GH}-0.06-50$, and $\mathrm{RH}-0.06-50$ films were investigated by monitoring the variations of temperature, when the films were exposed to the near infrared (NIR) light. As shown in Fig. 7a, the equilibrium temperature of the films was proportional to the output power of the light source. The photothermal conversion efficiency of the composite film with GO or RGO was much higher than that of pure HPC film. When compared to GOHPC film, the RGO-HPC film had relatively high conversion efficiency, which is consistent with the findings in the composite polymers and hydrogels. ${ }^{25,27}$ It was also found that the reduction time had no impact on the conversion efficiency of the composite films. There were two stages in the kinetic curves of the temperature of RGO-HPC film under the NIR light. In the initial stage, the temperature increased linearly with time and the increasing rate was independent on the output power. However, temperature in the subsequent plateau stage increased with the increase in the output power (Fig. 7b). The temperature of the $\mathrm{RH}-0.06-50$ film increased from $20{ }^{\circ} \mathrm{C}$ to $80-105{ }^{\circ} \mathrm{C}$ within $\sim 4 \mathrm{~s}$. After turning off the light, temperature decreased dramatically with initial decreasing rate of $17-50{ }^{\circ} \mathrm{C} \mathrm{s}^{-1}$.

As mentioned previously, the mechanical properties of the films varied with the relative humidity. Therefore, the mechanical properties of the films should respond to a $\mathrm{RH}$ change. As shown in Fig. 8a, the environmental $\mathrm{RH}$ was switched from $35 \%$ to $60 \%$, in which stress relaxation was performed to the films. At a constant $\mathrm{RH}$ of $35 \%$, the stress of the H-50 film followed the typical exponential relaxation (Fig. 8c). Before applying the $\mathrm{RH}$ change, the stress relaxation curves of HPC, GO-HPC, and RGO-HPC films also followed the exponential relaxation kinetics. When RH quickly increased from $35 \%$ to $60 \%$, maintained this value for a short, and then returned to $\mathrm{RH}$ of $35 \%$, the stress first decreased dramatically due to the absorption of water, and then returned to the exponential mode, indicating the recovery of the film structure due to the evaporation of water to equilibrate with the environment. This trend repeated for the subsequent cyclic stimuli, during which the stress gradually became negative with time, suggesting the increased sample length. This was because the absorbed water plasticized HPC matrices and swelled the films, thus the tensile stress became the compressive stress. This suggested that the stress drop after applying the stimuli was not only caused by the decrease of the film stiffness, but also the increase of film length. The stress drop increased with the time during which the film exposed to high $\mathrm{RH}$ vapour. After evaporation, the film became stiffer, but the film length
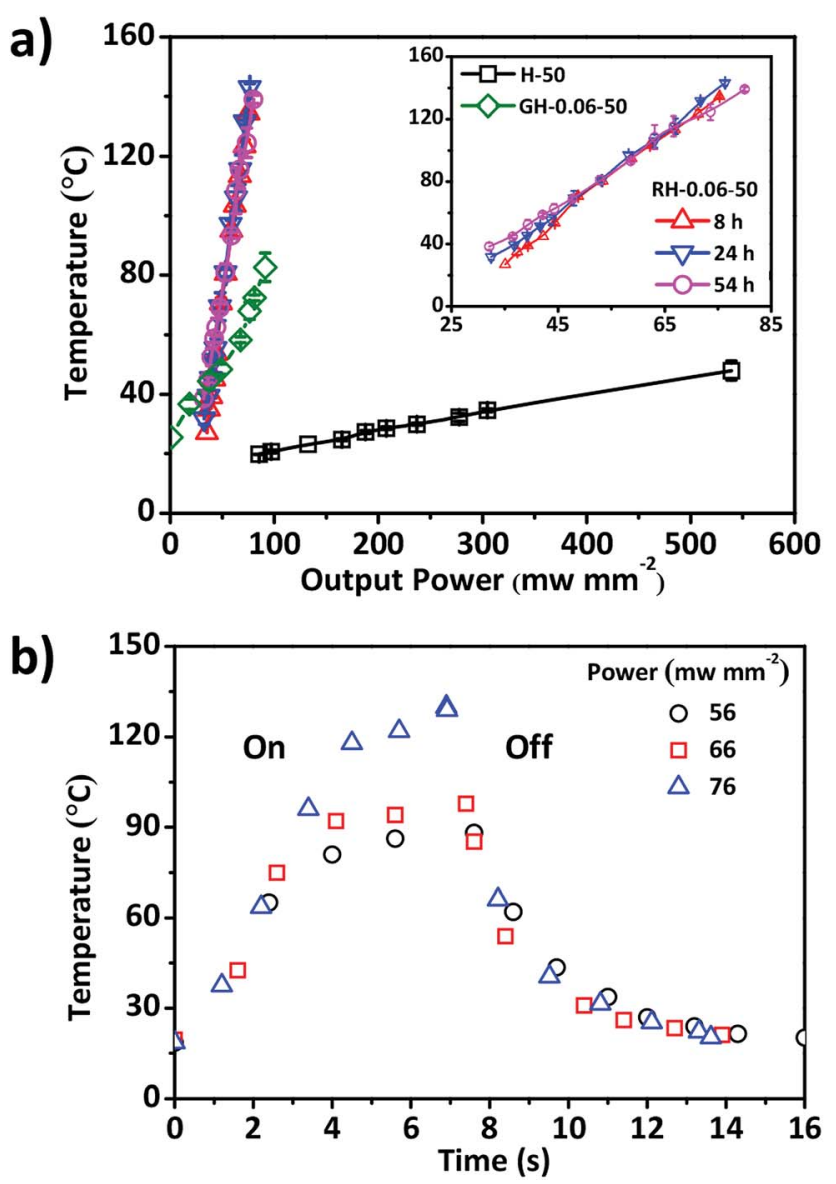

Fig. 7 (a) Equilibrium temperature of $\mathrm{H}-50, \mathrm{GH}-0.06-50$, and $\mathrm{RH}-$ 0.06-50 composite films as a function of output power of NIR light. The films were prepared by $254 \mathrm{~nm}$ light irradiation for different time. (b) Variation of the temperature of the $\mathrm{RH}-0.06-50$ film after turning on and turning off the NIR light. 
experienced plastic deformation cannot return to the original value. The averaged stress drop rate for HPC and GOHPC films was similar, while RGO-HPC films had the smallest stress drop rate due to the relatively higher hydrophobicity of RGO than HPC and GO. With more stimuli cycles applied to the films, the stress drop rate decreased because the swelling of the films reached equilibrium with time.

The mechanical properties of the composite films should be also responsive to NIR light owing to the photothermal effect of GO and RGO. As shown in Fig. 8b, pulse NIR light irradiation was applied to the films during the stress relaxation test. The stress of the pure HPC film followed the typical exponential relaxation and no response of the film to NIR light was observed. The stress of GO-HPC and RGO-HPC films first decreased upon the stimuli and increased a little until showing a plateau when the stimuli were removed (Fig. 8d). It was found that longer exposure time caused a larger stress drop, and the stress drop of the RGO-HPC film was larger than that of GO-HPC film, confirming that RGOHPC films have larger photothermal efficiency. It is straightforward to correlate the response with the photothermal effect mediated temperature rise that locally changed the surrounding $\mathrm{RH}$ of the composite films. A decrease in RH and consequently an increase in the stress upon NIR light irradiation should be expected. However, GO-HPC and RGO-HPC films showed stress drops when exposed to the NIR light. The stress also became negative after several pulse NIR light irradiation, indicating the increase in length of the sample probably due to the volume expansion of HPC matrices at high temperature. The little rise of dropped stress after withdrawing the NIR light should be associated with the slight recovery of the length due to the volume contraction of the film when the temperature was back to room temperature. This assumption was verified by the permanent buckling deformation of the RGO-HPC film after the irradiation under UV light. As shown in Fig. 8e, the film composite buckled upward to the irradiated side, which was irreversible after the irradiation. The permanent deformation stemmed from the structural rearrangement and volume expansion of the HPC matrices at high temperature. The responses of composite films to the humidity gradient and the light irradiation with controllable directions may endow the films with potential applications in sensing and actuation.
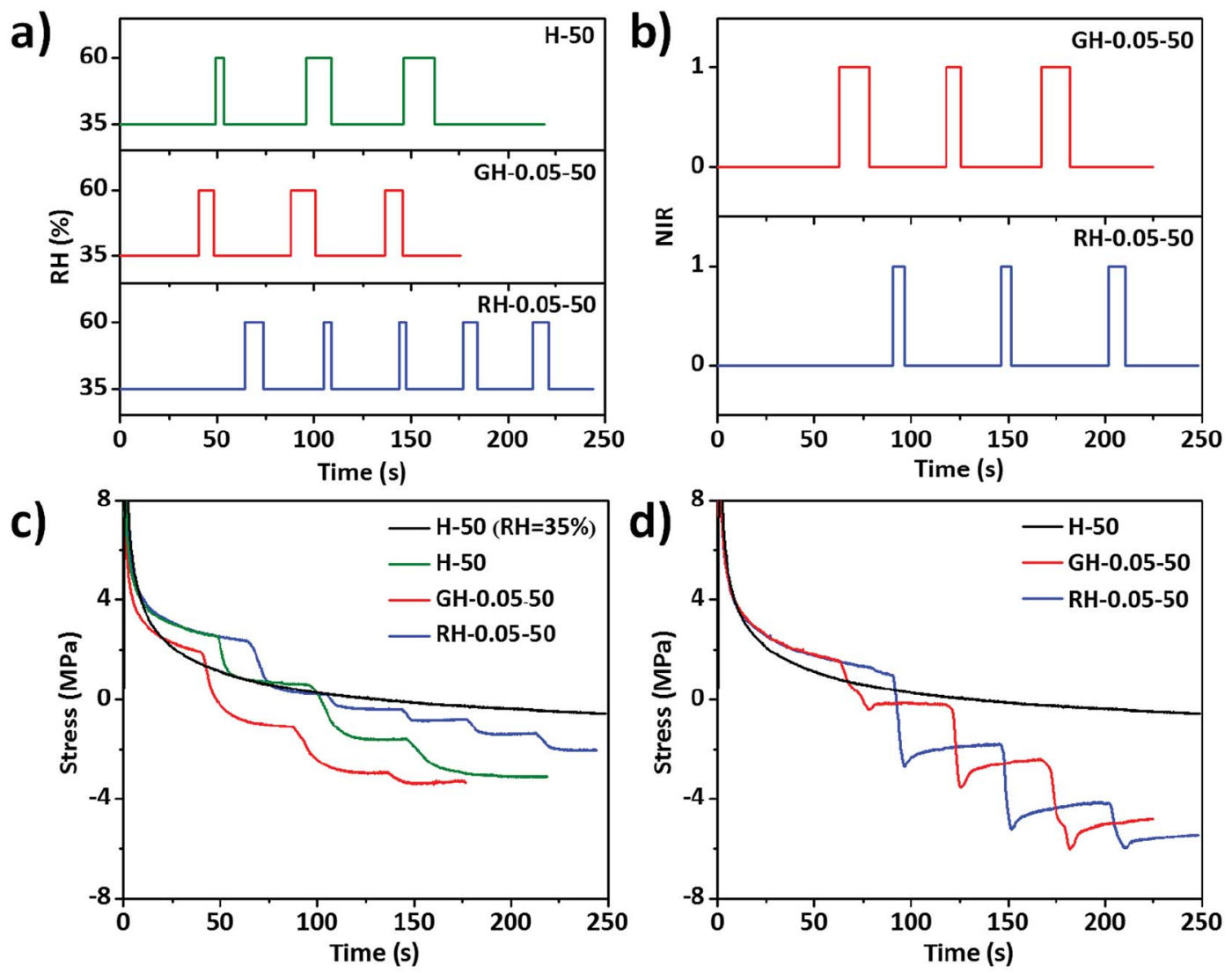

e)
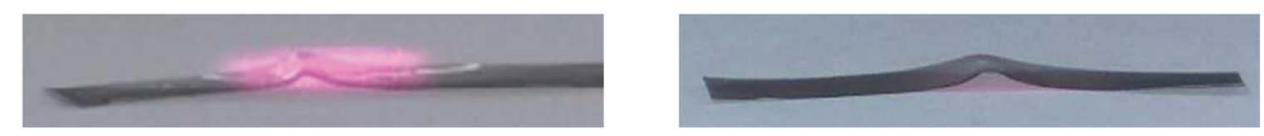

Fig. 8 Stress relaxation curves of composite films under cyclic humidity change (a and c) and $808 \mathrm{~nm}$ IR light irradiation at relative humidity of $35 \%$ ( $b$ and d). The film was elongated to $3 \%$ strain under a stretch rate of $40 \mathrm{~mm} \mathrm{~min}{ }^{-1}$ at room temperature. (e) $\mathrm{Photo}$ of a $\mathrm{RH}-0.05-50$ film during (left) and after (right) exposed to NIR light. 


\section{Conclusions}

In summary, we have fabricated anisotropic GO-HPC composite films by blade-coating and subsequent solvent evaporation. The incorporation of GO did not destroy the LC structure of HPC solution, yet improved the mechanical properties of the films to some extent. Increasing the relative humidity weakened the HPC films and decreased the mechanical anisotropy of the films, while incorporating GO can prevent the anisotropic properties being compromised. The incorporation of GO afforded the anisotropic composite film better response to humidity gradients; it exhibited continuous directional bending, flipping, and locomotion under a humidity gradient due to the excellent gas barrier property of GO that favoured the differential volume change at the top and bottom surfaces of the film. Besides the response to humidity gradient, the composite HPC film with GO or RGO showed irreversible volume expansion under NIR light irradiation due to the variation of the HPC matrix at high temperature because of the photothermal effect of GO and RGO. These anisotropic composite films with improved mechanical properties and multi-responsiveness have potential to construct sensors and actuators.

\section{Conflicts of interest}

There are no conflicts to declare.

\section{Acknowledgements}

We acknowledge the support of National Natural Science Foundation of China (51773179), Excellent Youth Foundation of Zhejiang Province of China (LR19E030002), Fundamental Research Funds for the Central Universities of China, and Zhejiang University K. P. Chao's High Technology Development Foundation.

\section{References}

1 S. Wang, A. Lu and L. Zhang, Recent advances in regenerated cellulose materials, Prog. Polym. Sci., 2016, 53, 169-206.

2 Y. H. Jung, T.-H. Chang, H. Zhang, C. Yao, Q. Zheng, V. W. Yang, H. Mi, M. Kim, S. J. Cho, D.-W. Park, H. Jiang, J. Lee, Y. Qiu, W. Zhou, Z. Cai, S. Gong and Z. Ma, Highperformance green flexible electronics based on biodegradable cellulose nanofibril paper, Nat. Commun., 2015, 6, 7170.

3 J. Song, C. Chen, S. Zhu, M. Zhu, J. Dai, U. Ray, Y. Li, Y. Kuang, Y. Li, N. Quispe, Y. Yao, A. Gong, U. H. Leiste, H. A. Bruck, J. Y. Zhu, A. Vellore, H. Li, M. L. Minus, Z. Jia, A. Martini, T. Li and L. Hu, Processing bulk natural wood into a high-performance structural material, Nature, 2018, 554, 224-228.

4 K. Zhu, H. Tu, P. Yang, C. Qiu, D. Zhang, A. Lu, L. Luo, F. Chen, X. Liu, L. Chen, Q. Fu and L. Zhang, Mechanically strong chitin fibers with nanofibril structure, biocompatibility, and biodegradability, Chem. Mater., 2019, 31, 2078-2087.

5 Y. Habibi, L. A. Lucia and O. J. Rojas, Cellulose nanocrystals: chemistry, self-assembly, and applications, Chem. Rev., 2010, 110, 3479-3500.

6 M. Zhu, Y. Wang, S. Zhu, L. Xu, C. Jia, J. Dai, J. Song, Y. Yao, Y. Wang, Y. Li, D. Henderson, W. Luo, H. Li, M. L. Minus, T. $\mathrm{Li}$ and $\mathrm{L}$. $\mathrm{Hu}$, Anisotropic, transparent films with aligned cellulose nanofibers, Adv. Mater., 2017, 29, 1606284.

7 K. M. O. Håkansson, A. B. Fall, F. Lundell, S. Yu, C. Krywka, S. V. Roth, G. Santoro, M. Kvick, L. P. Wittberg, L. Wågberg and L. D. Söderberg, Hydrodynamic alignment and assembly of nanofibrils resulting in strong cellulose filaments, Nat. Commun., 2014, 5, 4018.

8 M. Wang, X. Tian, R. H. A. Ras and O. Ikkala, Sensitive Humidity-driven reversible and bidirectional bending of nanocellulose thin films as bio-inspired actuation, $A d v$. Mater. Interfaces, 2015, 2, 1500080.

9 W. Yang, Z. Zhao, K. Wu, R. Huang, T. Liu, H. Jiang, F. Chen and $\mathrm{Q}$. $\mathrm{Fu}$, Ultrathin flexible reduced graphene oxide/ cellulose nanofiber composite films with strongly anisotropic thermal conductivity and efficient electromagnetic interference shielding, J. Mater. Chem. C, 2017, 5, 3748-3756.

10 D. N.-S. Hon, Cellulose and its derivatives: structures, reactions, and medical uses, Marcel Dekker, New York, 1996.

11 S. Fortin and G. Charlet, Phase diagram of aqueous solutions of (hydroxypropyl)cellulose, Macromolecules, 1989, 22, 22862292.

12 X. Y. Lin, Z. J. Wang, P. Pan, Z. L. Wu and Q. Zheng, Monodomain hydrogels prepared by shear-induced orientation and subsequent gelation, RSC Adv., 2016, 6, 95239-95245.

13 M. H. Godinho, D. G. Gray and P. Pieranski, Revisiting (hydroxypropyl) cellulose (HPC)/water liquid crystalline system, Liq. Cryst., 2017, 44, 2108-2120.

14 Y. Geng, P. L. Almeida, S. N. Fernandes, C. Cheng, P. PalffyMuhoray and M. H. Godinho, A cellulose liquid crystal motor: a steam engine of the second kind, Sci. Rep., 2013, 3, 1028.

15 S. N. Fernandes, Y. Geng, S. Vignolini, B. J. Glover, A. C. Trindade, J. P. Canejo, P. L. Almeida, P. Brogueira and M. H. Godinho, Structural color and iridescence in transparent sheared cellulosic films, Macromol. Chem. Phys., 2013, 214, 25-32.

16 C. Echeverria, L. E. Aguirre, E. G. Merino, P. L. Almeida and M. H. Godinho, Carbon nanotubes as reinforcement of cellulose liquid crystalline responsive networks, ACS Appl. Mater. Interfaces, 2015, 7, 21005-21009.

$17 \mathrm{Z}$. Xu and C. Gao, Aqueous liquid crystals of graphene oxide, ACS Nano, 2011, 5, 2908-2915.

18 H.-P. Cong, P. Wang and S.-H. Yu, Highly elastic and superstretchable graphene oxide/polyacrylamide hydrogels, Small, 2014, 10, 448-453.

19 Q. Cheng, M. Wu, M. Li, L. Jiang and Z. Tang, Ultratough artificial nacre based on conjugated cross-linked graphene oxide, Angew. Chem., Int. Ed., 2013, 52, 3750-3755. 
20 W. Cui, M. Li, J. Liu, B. Wang, C. Zhang, L. Jiang and Q. Cheng, A strong integrated strength and toughness artificial nacre based on dopamine cross-linked graphene oxide, ACS Nano, 2014, 8, 9511-9517.

21 H.-P. Cong, P. Wang and S.-H. Yu, Stretchable and selfhealing graphene oxide-polymer composite hydrogels: a dual-network design, Chem. Mater., 2013, 25, 3357-3362.

22 S. Wan, J. Peng, Y. Li, H. Hu, L. Jiang and Q. Cheng, Use of synergistic interactions to fabricate strong, tough, and conductive artificial nacre based on graphene oxide and chitosan, ACS Nano, 2015, 9, 9830-9836.

23 Y.-H. Yang, L. Bolling, M. A. Priolo and J. C. Grunlan, Super gas barrier and selectivity of graphene oxide-polymer multilayer thin films, Adv. Mater., 2013, 25, 503-508.

24 P. Xiao, N. Yi, T. Zhang, Y. Huang, H. Chang, Y. Yang, Y. Zhou and Y. Chen, Construction of a fish-like robot based on high performance graphene/PVDF bimorph actuation materials, Adv. Sci., 2016, 3, 1500438.

25 D. Kim, H. S. Lee and J. Yoon, Highly bendable bilayer-type photo-actuators comprising of reduced graphene oxide dispersed in hydrogels, Sci. Rep., 2016, 6, 20921.

26 J. He, P. Xiao, J. Zhang, Z. Liu, W. Wang, L. Qu, Q. Ouyang, X. Wang, Y. Chen and T. Chen, Highly efficient actuator of graphene/polydopamine uniform composite thin film driven by moisture gradients, Adv. Mater. Interfaces, 2016, 3, 1600169.

27 M. Ji, N. Jiang, J. Chang and J. Sun, Near-infrared lightdriven, highly efficient bilayer actuators based on polydopamine-modified reduced graphene oxide, $A d v$. Funct. Mater., 2014, 24, 5412-5419.

28 C.-H. Zhu, Y. Lu, J. Peng, J.-F. Chen and S.-H. Yu, Photothermally sensitive poly( $N$-isopropylacrylamide $) /$ graphene oxide nanocomposite hydrogels as remote lightcontrolled liquid microvalves, Adv. Funct. Mater., 2012, 22, 4017-4022.
29 Y. Ge, R. Cao, S. Ye, Z. Chen, Z. Zhu, Y. Tu, D. Ge and X. Yang, A bio-inspired homogeneous graphene oxide actuator driven by moisture gradients, Chem. Commun., 2018, 54, 31263129.

$30 \mathrm{Z}$. Xu, Y. Zhang, P. Li and C. Gao, Strong, conductive, lightweight, neat graphene aerogel fibers with aligned pores, ACS Nano, 2012, 6, 7103-7113.

31 L. Kou and C. Gao, Bioinspired design and macroscopic assembly of poly(vinyl alcohol)-coated graphene into kilometers-long fibers, Nanoscale, 2013, 5, 4370-4378.

32 B. Ernst and P. Navard, Band textures in mesomorphic (hydroxypropyl) cellulose solutions, Macromolecules, 1989, 22, 1419-1422.

33 P. Navard, Formation of band textures in hydroxypropylcellulose liquid crystals, J. Polym. Sci., Part B: Polym. Phys., 1986, 24, 435-442.

34 B. Zhang, K. Li and J. Zhao, Regularity analysis of wrinkles under the action of capillary force in an annular thin film, Sci. China: Phys., Mech. Astron., 2014, 57, 1574-1580.

35 L. Zhang, H. Liang, J. Jacob and P. Naumov, Photogated humidity-driven motility, Nat. Commun., 2015, 6, 7429.

36 S. Liang, X. Qiu, J. Yuan, W. Huang, X. Du and L. Zhang, Multiresponsive kinematics and robotics of surfacepatterned polymer film, ACS Appl. Mater. Interfaces, 2018, 10, 19123-19132.

37 M. Ma, L. Guo, D. G. Anderson and R. Langer, Bio-inspired polymer composite actuator and generator driven by water gradients, Science, 2013, 339, 186-189.

38 E. Zhang, T. Wang, W. Hong, W. Sun, X. Liu and Z. Tong, Infrared-driving actuation based on bilayer graphene oxide-poly( $N$-isopropylacrylamide) nanocomposite hydrogels, J. Mater. Chem. A, 2014, 2, 15633-15639.

39 Z. J. Wang, C. Y. Li, X. Y. Zhao, Z. L. Wu and Q. Zheng, Thermo- and photo-responsive composite hydrogels with programmed deformations, J. Mater. Chem. B, 2019, 7, 1674-1678. 